PROCEEDINGS OF THE

AMERICAN MATHEMATICAL SOCIETY

Volume 140, Number 2, February 2012, Pages 553-560

S 0002-9939(2011)10998-5

Article electronically published on June 2, 2011

\title{
THE HULL OF RUDIN'S KLEIN BOTTLE
}

\author{
JOHN T. ANDERSON
}

(Communicated by Franc Forstneric)

\begin{abstract}
In 1981 Walter Rudin exhibited a totally real embedding of the Klein bottle into $\mathbb{C}^{2}$. We show that the polynomially convex hull of Rudin's Klein bottle contains an open subset of $\mathbb{C}^{2}$. We also describe another totally real Klein bottle in $\mathbb{C}^{2}$ whose hull has topological dimension equal to three.
\end{abstract}

\section{INTRODUCTION}

A real submanifold $M$ of complex Euclidean space $\mathbb{C}^{n}$ is said to be totally real if, at each point $p \in M$, the tangent space $T_{p}(M)$ to $M$ (with the usual identifications of $T_{p}(M)$ with a subspace of $\mathbb{R}^{2 n}$ ) contains no nontrivial complex subspace. Among the real surfaces, only the torus and connected sums of an odd number of Klein bottles admit totally real embeddings into $\mathbb{C}^{2}$ (see for example [5]). The first explicit example of a totally real embedding of the Klein bottle in $\mathbb{C}^{2}$ was given by Walter Rudin in [10. Section 20 of $[3]$ describes a family of immersions of the Klein bottle into $\mathbb{C}^{2}$ that includes Rudin's embedding.

For a compact subset $Y$ of $\mathbb{C}^{n}$, the polynomially convex hull of $Y$ is defined by

$$
\widehat{Y}=\left\{z \in \mathbb{C}^{n}:|P(z)| \leq\|P\|_{Y} \text { for all holomorphic polynomials } P\right\},
$$

where $\|P\|_{Y}=\max \{|P(\zeta)|: \zeta \in Y\}$. The set $\widehat{Y}$ may be identified with the maximal ideal space of the algebra $P(Y)$ of uniform limits of holomorphic polynomials on $Y$. We say that $Y$ is polynomially convex if $\widehat{Y}=Y$. If $M$ is a compact real submanifold of $\mathbb{C}^{n}$ of dimension $n$, then $M$ is not polynomially convex (see 11], Corollary 2.3.5). For orientable $M$ this follows from a result of A. Browder; for nonorientable $M$ it is due to T. Duchamp and E. L. Stout. In fact, a result of H. Alexander implies that the topological dimension of $\widehat{M} \backslash M$ must be at least $n+1$ (11, Corollary 2.3.18). One would like to "explain" these topological results analytically, say by constructing holomorphic maps of the open unit disk $\mathbb{D}$ into $\widehat{M} \backslash M$ with $\partial \mathbb{D}$ mapped to $M$. By the maximum principle, the image of $\mathbb{D}$ under any such map lies in $\widehat{M}$.

For a submanifold $M$ of $\mathbb{C}^{n}$ which is not totally real, a technique due to E. Bishop and since refined by a number of authors allows, under certain nondegeneracy conditions, construction of such analytic disks with boundaries in $M$ near a point of complex tangency. In the case of totally real manifolds, of course, this approach is not possible. Nevertheless, it is still often possible to find holomorphic mappings

Received by the editors November 20, 2010.

2010 Mathematics Subject Classification. Primary 32E20; Secondary 32V40.

Key words and phrases. Polynomial hull, Klein bottle.

(C)2011 American Mathematical Society Reverts to public domain 28 years from publication 
of the disk into $\mathbb{C}^{n}$ with boundaries on $M$. In this paper we will use a theorem of J. Wermer [12] to produce enough analytic disks with boundaries attached to Rudin's Klein bottle so that their interiors fill an open subset of $\mathbb{C}^{2}$. This method was also used in 2 to determine the hull of a certain totally real embedding of the threesphere into $\mathbb{C}^{3}$ constructed by Ahern and Rudin. In [6], Forstnerič showed, using his deep results on perturbing analytic disks with boundaries in a real submanifold, that the hull of Rudin's Klein bottle contains an open set. Our methods are more elementary and give a more explicit description.

In section 2 we recall Rudin's construction and introduce a covering of Rudin's Klein bottle $K$ by a totally real torus. In section 3 we determine exactly the polynomially convex hull of the covering torus and conclude that the hull of $K$ contains an open subset of $\mathbb{C}^{2}$. In section 4 we modify Rudin's example to produce a totally real Klein bottle in $\mathbb{C}^{2}$ whose hull has topological dimension three (which, by Alexander's result, is as small as possible).

The general problem of attaching analytic disks (or more general holomorphic sets) to totally real submanifolds of complex manifolds has been studied intensively. The paper [4] gives some history of this problem and contains results on attaching Riemann surfaces to totally real tori. The author is unaware of any general results on producing analytic disks with boundaries in a Klein bottle.

\section{Rudin's Klein Bottle And A COVERING}

We first describe the construction given in $[10$.

Define $F=\left(F_{1}, F_{2}\right): \mathbb{R}^{2} \rightarrow \mathbb{C}^{2}$ by

$$
F(\theta, \phi):=\left(e^{2 i \theta} k^{2}(\phi), e^{i \theta} k(\phi) h(\phi)\right),
$$

where $k, h$ are smooth, $2 \pi$-periodic functions on the real line with

(i) $k$ real-valued, even, everywhere positive and strictly monotone on $[-\pi, 0)$;

(ii) $h$ complex-valued, odd and $h(\phi) \neq 0$ for $\phi \in(0, \pi)$;

(iii) $h^{\prime}$ nonzero everywhere.

Let $R=[0, \pi) \times[-\pi, \pi)$. It is easy to check that under assumptions (i), (ii), and (iii) we have

(1) $F(\theta, \phi+2 \pi)=F(\theta, \phi)=F(\theta+\pi,-\phi)$;

(2) $F$ is one-to-one on $R$;

(3) $d F_{1} \wedge d F_{2} \neq 0$ on $R$.

Properties (1) - (3) imply that $F(R)$ is a smooth, totally real submanifold of $\mathbb{C}^{2}$ homeomorphic to a Klein bottle. In Rudin's construction,

$$
k(\phi):=a+b \cos (\phi) \text { where } a>b>0, \text { and } h(\phi):=\sin (\phi)+i \sin (2 \phi) .
$$

Henceforth we assume that $k, h$ are as in (2.1), and we set $K=F(R)$.

For fixed $\phi$, the map $\zeta \in \mathbb{D} \mapsto\left(\zeta^{2} k^{2}(\phi), \zeta k(\phi) h(\phi)\right)$ is clearly an analytic disk with boundary in $K$, but as we shall see the union of this one-parameter family of disks does not fill out $\widehat{K}$.

Next we consider the map $\widetilde{F}=\left(\widetilde{F}_{1}, \widetilde{F}_{2}\right): \mathbb{R}^{2} \mapsto \mathbb{C}^{2}$ defined by

$$
\widetilde{F}(\theta, \phi)=\left(e^{i \theta} k(\phi), h(\phi)\right):=(z(\theta, \phi), w(\theta, \phi)) .
$$


Let $\widetilde{R}=[-\pi, \pi) \times[-\pi, \pi) \subset \mathbb{C}^{2}$. Then it easy to verify that

(1) $\widetilde{F}(\theta+2 \pi, \phi)=\widetilde{F}(\theta, \phi)=\widetilde{F}(\theta, \phi+2 \pi)$;

(2) $\widetilde{F}$ is one-to-one on $\widetilde{R}$;

(3) $d \widetilde{F}_{1} \wedge d \widetilde{F}_{2} \neq 0$ on $\widetilde{R}$.

Properties (1) - (3) imply that $T:=\widetilde{F}(\widetilde{R})$ is a smooth, totally real submanifold of $\mathbb{C}^{2}$ homeomorphic to a torus. Set

$$
Q(z, w):=\left(z^{2}, z w\right) .
$$

It is not hard to check that $Q(T)=K$ and that if $q=F(\theta, \phi) \in K$, then $Q^{-1}(\{q\})$ consists of the two points $\tilde{F}(\theta, \phi)$ and $\tilde{F}(\theta+\pi,-\phi)$. We will determine exactly the polynomially convex hull $\widehat{T}$ of $T$ and show that it contains an open subset of $\mathbb{C}^{2}$. Since $Q$ is a polynomial map, $Q(\widehat{T}) \subset \hat{K}$, and therefore (note $Q$ has a nonvanishing Jacobian determinant for $z \neq 0) \widehat{K}$ contains an open subset of $\mathbb{C}^{2}$. However, polynomial maps do not necessarily preserve polynomially convex hulls: consider, for example, the image under the map $Q$ of $\Gamma:=\left\{\left(e^{i \theta}, e^{-i \theta}\right): \theta \in[-\pi, \pi)\right\}$. The circle $\Gamma$ is polynomially convex, but $Q(\Gamma)=\left\{\left(e^{2 i \theta}, 1\right): \theta \in[-\pi, \pi)\right\}$ is not. We have been unable to prove that $Q(\widehat{T})=\widehat{K}$.

\section{The Hull of $T$}

As is well known, in considering analytic disks with boundaries on a compact set $Y \subset \mathbb{C}^{n}$ as a way of exhibiting points in the hull of $Y$, we need not insist that the boundary of the image is attached smoothly to $Y$. Indeed, if $\Phi: \mathbb{D} \mapsto \mathbb{C}$ is holomorphic and bounded and if $\Phi^{*}\left(e^{i \theta}\right):=\lim _{r \uparrow 1} \Phi\left(r e^{i \theta}\right) \in Y$ for almost all $\theta \in[0,2 \pi)$, then the maximum principle for bounded holomorphic functions implies that $\Phi(\mathbb{D}) \subset \widehat{Y}$. We say that $\Phi$ (more precisely, its image) is an $H^{\infty}$-disk with boundary in $Y$.

With $h, k$ as in (2.1), set $\gamma=\{h(\phi): \phi \in[-\pi, \pi)\}$. The figure-eight curve $\gamma$ has a double point at the origin ( since $h(0)=h(-\pi)=0$ ), while $h$ maps $[-\pi, 0) \cup(0, \pi)$ one-to-one onto $\gamma \backslash\{0\}$. Let $\Omega$ be the region bounded by $\gamma ; \Omega$ is symmetric with respect to the origin. For $\phi \in[-\pi, \pi)$ we set

$$
\Gamma_{\phi}=\{(z, w) \in T: w=h(\phi)\}=\left\{\left(e^{i \theta} k(\phi), h(\phi)\right): \theta \in[-\pi, \pi)\right\},
$$

and let $D_{\phi}$ be the (closed) $H^{\infty}$-disk with boundary $\Gamma_{\phi}$, i.e.,

$$
D_{\phi}=\{(z, h(\phi)):|z| \leq k(\phi)\} .
$$

If $\phi \neq 0, D_{\phi} \cap T=\Gamma_{\phi}$, while $D_{0} \cap T=\Gamma_{0} \cup \Gamma_{\pi}=\{(z, 0):|z|=a \pm b\}$. We consider the Dirichlet problem

$$
\triangle u=0 \text { in } \Omega, u=\log \left(k \circ h^{-1}\right) \text { on } \gamma \backslash\{0\} .
$$

Note that $k \circ h^{-1}$ is continuous, bounded and bounded away from zero on $\gamma \backslash\{0\}$. There thus exists (see [9], Corollary 4.2.6 and its proof) a unique real-valued function $u \in L^{\infty}(\Omega)$, continuous on $\bar{\Omega} \backslash\{0\}$, satisfying (3.1). In fact, $u$ is continuously differentiable on $\bar{\Omega} \backslash\{0\}$ (see [7, chapter II, section 4). The fact that $h$ is odd and $k$ is even together with uniqueness of the solution to (3.1) implies that $u(w)=u(-w)$ for all $w \in \Omega$.

Let $v$ be a harmonic conjugate for $u$ in $\Omega$. We may construct $v$ on the simply connected region $\Omega^{+}=\Omega \cap\{w \in \mathbb{C}: \operatorname{Re}(w)>0\}$ and extend $v$ to $\Omega \backslash \Omega^{+}$by setting $v(w)=v(-w)$. Since $u$ is continuously differentiable on $\bar{\Omega} \backslash\{0\}, v$ is continuous 
on $\bar{\Omega} \backslash\{0\}$. (See for example [8], p. 18. The result proven there for the disk may be transferred to $\Omega^{+}$by means of a conformal map. Since $\gamma \backslash\{0\}$ is real analytic, any conformal map $\beta$ from $\mathbb{D}$ to $\Omega^{+}$extends holomorphically to a neighborhood of each point in $\partial \mathbb{D} \backslash \beta^{-1}(0)$, by Schwarz reflection.) Define $g=\exp (u+i v)$. Then $g \in H^{\infty}(\Omega)$, and $g$ extends continuously to $\gamma \backslash\{0\}$ with

$$
\left|g\left(w_{0}\right)\right|=k \circ h^{-1}\left(w_{0}\right), w_{0} \in \gamma \backslash\{0\} .
$$

We may now state the description of $\widehat{T}$ :

\section{Theorem 3.1.}

$$
\widehat{T}=\{(z, w): w \in \bar{\Omega} \backslash\{0\},|z| \leq|g(w)|\} \cup D_{0},
$$

and therefore $\widehat{T}$ contains an open subset of $\mathbb{C}^{2}$.

As remarked at the end of the previous section, Theorem 3.1 immediately gives the following information about $\widehat{K}$ :

Corollary 1. With $Q(z, w)=\left(z^{2}, z w\right), Q(\widehat{T}) \subset \widehat{K}$, and therefore $\widehat{K}$ contains an open subset of $\mathbb{C}^{2}$.

The proof of Theorem 3.1 will be divided into three lemmas. Note that since $\bar{\Omega}$ is polynomially convex, $(z, w) \in \widehat{T}$ implies $w \in \bar{\Omega}$. We begin by describing the points in the hull for which $w \in \gamma$.

\section{Lemma 3.2.}

$$
\{(z, w) \in \widehat{T}: w \in \gamma\}=\bigcup_{\phi \in(-\pi, \pi)} D_{\phi}
$$

Proof. Each $D_{\phi}$ is contained in $\widehat{T}$, so the set on the right-hand side of (3.3) is contained in the set on the left-hand side. The proof of the opposite inclusion is essentially the same as that given in [1] for the first claim in the proof of Theorem 1 of that paper. For convenience we reproduce the argument. Suppose $p=\left(z_{0}, w_{0}\right) \in$ $\widehat{T}$ and $w_{0}=h(\phi)$. If $p \notin D_{\phi}$, then $\left|z_{0}\right|>k(\phi)$. Every point of $\gamma$ is a peak point for the algebra $P(\bar{\Omega})$, so we can choose $f \in P(\bar{\Omega})$ with $f\left(w_{0}\right)=1$ and $|f|<1$ on $\gamma \backslash\left\{w_{0}\right\}$. For each integer $n \geq 0$, the function $(z, w) \mapsto z f^{n}(w)$ belongs to $P(T)$. If $\mu$ is a representing measure for $p$ for the algebra $P(T)$ with support in $T$ (see for example [11, p. 10), then

$$
\left|\int_{T} z f^{n}(w) d \mu(z, w)\right|=\left|z_{0} f^{n}\left(w_{0}\right)\right|=\left|z_{0}\right| .
$$

However, taking the limit as $n \rightarrow \infty$ in (3.4) gives

$$
\left|\int_{\Gamma_{\phi}} z d \mu(z, w)\right|=\left|z_{0}\right|>k(\phi),
$$

contradicting the fact that $|z|=k(\phi)$ on $\Gamma_{\phi}$.

Since $|g(h(\phi))|=k(\phi)$ for $\phi \notin\{-\pi, 0\}$ and $|z| \leq k(\phi)$ if $(z, w) \in D_{\phi}$, we can express the result of Lemma 3.2 as follows:

$$
\{(z, w) \in \widehat{T}: w \in \gamma\}=\{(z, w): w \in \gamma \backslash\{0\},|z| \leq|g(w)|\} \cup D_{0} .
$$


Next we show:

\section{Lemma 3.3.}

$$
\{(z, w): w \in \Omega,|z| \leq|g(w)|\} \subset \widehat{T} \text {. }
$$

Proof. It suffices to show that

$$
w \in \Omega,|z|=|g(w)| \Rightarrow(z, w) \in \widehat{T}
$$

for then the map $\zeta \in \mathbb{D} \mapsto(\zeta|g(w)|, w)$ is (for each fixed $w \in \Omega$ ) an $H^{\infty}$-disk with boundary in $\widehat{T}$, hence $(\zeta|g(w)|, w) \subset \widehat{T}$ for all $\zeta \in \mathbb{D}$, establishing (3.6). Moreover, $T$ is invariant under $(z, w) \rightarrow(z,-w)$, so the same is true of $\widehat{T}$. Since $\log |g|=u$ is even, it therefore suffices to prove (3.7) for $w \in \Omega^{+}$. Let $\psi$ be a conformal map of the unit disk onto $\Omega^{+}$. Then $\psi$ extends to a homeomorphism of $\overline{\mathbb{D}}$ with $\overline{\Omega^{+}}$. We may assume that $\psi(1)=0$. Then for $\lambda_{0} \in \partial \mathbb{D} \backslash\{1\}$,

$$
\lim _{\lambda \in \mathbb{D} \rightarrow \lambda_{0}}|g \circ \psi(\lambda)|=k \circ h^{-1} \circ \psi\left(\lambda_{0}\right)=k(\phi),
$$

where $\phi=h^{-1} \circ \psi\left(\lambda_{0}\right)$. Therefore, for any $\tau \in[-\pi, \pi)$,

$$
\lim _{\lambda \in \mathbb{D} \rightarrow \lambda_{0}}\left(e^{i \tau} g \circ \psi(\lambda), \psi(\lambda)\right)=\left(e^{i \tau} e^{i \sigma} k(\phi), h(\phi)\right)=\widetilde{F}(\tau+\sigma, \phi)
$$

for some $\sigma \in[-\pi, \pi)$, hence $\lim _{\lambda \in \mathbb{D} \rightarrow \lambda_{0}}\left(e^{i \tau} g \circ \psi(\lambda), \psi(\lambda)\right) \in T$; i.e., the map $\lambda \in$ $\mathbb{D} \mapsto\left(e^{i \tau} g \circ \psi(\lambda), \psi(\lambda)\right)$ is an $H^{\infty}$-disk with boundary in $T$. Therefore, $\left(e^{i \tau} g \circ\right.$ $\psi(\lambda), \psi(\lambda)) \in \widehat{T}$ for all $\lambda \in \mathbb{D}$, implying $\left(e^{i \tau} g(w), w\right) \in \widehat{T}$ for all $w \in \Omega^{+}$. This establishes (3.7) and completes the proof.

Finally, we have

Lemma 3.4.

$$
\{(z, w) \in \widehat{T}: w \in \Omega\} \subset\{(z, w): w \in \Omega,|z| \leq|g(w)|\} .
$$

Proof. We make use of a theorem of John Wermer [12]: Let A be a uniform algebra on a compact Hausdorff space $X$, and let $\mathcal{M}_{A}$ denote the maximal ideal space of A. Fix functions $\pi, f \in A$, and let $\hat{\pi}, \hat{f}$ denote the Gelfand transforms of $\pi, f$ respectively. Set

$$
\psi(\zeta)=\log \left(\max \left\{|\hat{f}(p)|: p \in \mathcal{M}_{A} \text { and } \hat{\pi}(p)=\zeta\right\} .\right.
$$

Then $\psi$ is subharmonic on any open subset $U$ of $\mathbb{C} \backslash \pi(X)$ such that $\hat{\pi}^{-1}(U)$ is nonempty.

Taking $X=T, A=P(T)$ (so $\left.\mathcal{M}_{A}=\widehat{T}\right), f(z, w)=z$ and $\pi(z, w)=w$, Wermer's Theorem implies that

$$
\psi(w)=\log (\max \{|z|:(z, w) \in \widehat{T}\})
$$

is subharmonic on $\Omega$. We claim that if $w_{0} \in \gamma \backslash\{0\}$,

$$
\limsup _{w \in \Omega \rightarrow w_{0}} \psi(w)-\log |g(w)| \leq 0
$$

If (3.8) fails, then (using the fact that $|g|$ is continuous on $\bar{\Omega} \backslash\{0\}$ ) there exists $\epsilon>0$ and a sequence $w_{n} \in \Omega$ converging to $w_{0}$ with $\psi\left(w_{n}\right)>\log \left|g\left(w_{0}\right)\right|+\epsilon$ for all $n$. Choose $z_{n}$ so that $\left(z_{n}, w_{n}\right) \in \widehat{T}$ and $\log \left|z_{n}\right|=\psi\left(w_{n}\right)$. By passing to a subsequence, 
we may assume that $\left(z_{n}, w_{n}\right)$ converges to $\left(z_{0}, w_{0}\right) \in \widehat{T}$, for some $z_{0}$. By (3.5), $\log \left|z_{0}\right| \leq \log \left|g\left(w_{0}\right)\right|$, and so

$$
\log \left|z_{n}\right|=\psi\left(w_{n}\right)>\log \left|g\left(w_{0}\right)\right|+\epsilon \geq \log \left|z_{0}\right|+\epsilon
$$

for all $n$. However, $\log \left|z_{n}\right|$ converges to $\log \left|z_{0}\right|$, contradicting (3.9) and proving (3.8). Since $g$ is nonvanishing in $\Omega, \log |g|$ is harmonic and $\psi-\log |g|$ is subharmonic on $\Omega$. By the maximum principle for subharmonic functions bounded above on $\Omega$ (see 9], Theorem 3.6.9), (3.8) implies that $\psi(w)-\log |g(w)| \leq 0$ for all $w \in \Omega$. Since we also have by Lemma $3.3 \psi(w) \geq \log |g(w)|, \psi=\log |g|$ on $\Omega$. Therefore, if $(z, w) \in \widehat{T}$ and $w \in \Omega$,

$$
|z| \leq e^{\psi(w)}=|g(w)|
$$

This completes the proof of Lemma 3.4

Combining (3.5) with Lemmas 3.3 and 3.4 we obtain

$$
\widehat{T}=\{(z, w): w \in \bar{\Omega} \backslash\{0\},|z| \leq|g(w)|\} \cup D_{0} .
$$

Since $g$ is nonvanishing on $\Omega$, the set $\{(z, w): w \in \Omega,|z|<|g(w)|\}$ is open and contained in $\widehat{T}$. This completes the proof of Theorem 3.1 .

Remark 3.5. In Theorem 3.1 we have described the hull of $T$ as a union of "horizontal" disks $|z| \leq|g(w)|$. The proof of Lemma 3.3 shows that we can also write the hull (over $\Omega$ ) as a union of "vertical" disks $w \in \Omega \mapsto(\zeta g(w), w)$, where $\zeta \in \overline{\mathbb{D}}$. When $|\zeta|=1$, these disks are attached smoothly to $T$ except at $w=0$. The cluster set of each such disk at $w=0$ lies in $\{(z, 0): a-b \leq|z| \leq a+b\}$.

\section{A totally Real Klein bottle With a three-Dimensional hull}

Consider the map $\mathcal{F}=\left(\mathcal{F}_{1}, \mathcal{F}_{2}\right)$ defined by

$$
\mathcal{F}(\theta, \phi)=\left(e^{2 i \theta} k^{2}(\phi), e^{-i \theta} \frac{h(\phi)}{k(\phi)}\right)
$$

with $k, h$ as in (2.1). As in section 2 , we set $R=[0, \pi) \times[-\pi, \pi)$. It is straightforward to check the properties:

(1) $\mathcal{F}(\theta, \phi+2 \pi)=\mathcal{F}(\theta, \phi)=\mathcal{F}(\theta+\pi,-\phi)$;

(2) $\mathcal{F}$ is one-to-one on $R$;

(3) $d \mathcal{F}_{1} \wedge d \mathcal{F}_{2} \neq 0$ on $R$.

Therefore $\mathcal{K}=\mathcal{F}(R)$ is a totally real Klein bottle. We will give an explicit description of $\widehat{\mathcal{K}}$ and show that it has topological dimension equal to three. In this case we are able to dispense with the device of the covering torus. Since the methods are otherwise similar to those in section 3, we will omit some of the details.

Note that if $(z, w)=\mathcal{F}(\theta, \phi) \in \mathcal{K}$, then $z w^{2}=h^{2}(\phi)$. Set

$$
c:=\left\{h^{2}(\phi): \phi \in[-\pi, \pi)\right\} .
$$

The curve $c$ is the image under $\lambda \mapsto \lambda^{2}$ of the curve $\gamma$ appearing in section 3 ; $c$ is a closed curve with a cusp at $\lambda=0$, bounding a bounded simply connected region $O$. If $h(\phi) \neq 0$, i.e., $\phi \notin\{-\pi, 0\}$, then $h^{2}\left(\phi_{1}\right)=h^{2}\left(\phi_{2}\right)$ implies $\phi_{1}= \pm \phi_{2}$. We set

$$
\mathcal{C}_{\phi}=\left\{(z, w) \in \mathcal{K}: z w^{2}=h^{2}(\phi)\right\} .
$$

We may write for $\phi \notin\{-\pi, 0\}$,

$$
\mathcal{C}_{\phi}=\{F(\theta, \phi): \theta \in[0, \pi)\} \cup\{F(\theta,-\phi): \theta \in[0, \pi)\}=\{F(\theta, \phi): \theta \in[-\pi, \pi)\},
$$


where the second equality uses the fact that $\mathcal{F}(\theta, \phi)=\mathcal{F}(\theta+\pi,-\phi)$. It is easily verified that if $\phi \notin\{-\pi, 0\}$, the curve $\mathcal{C}_{\phi}$ is polynomially convex. Note that $\mathcal{C}_{0}=$ $\left\{(z, 0):|z|=(a \pm b)^{2}\right\}=\mathcal{C}_{-\pi}$, and so $\widehat{\mathcal{C}_{-\pi}}=\widehat{\mathcal{C}_{0}}=\mathcal{D}_{0}:=\left\{(z, 0):|z| \leq(a+b)^{2}\right\}$.

We let $u$ be the solution to the Dirichlet problem

$$
\triangle u=0 \text { in } O, u\left(h^{2}(\phi)\right)=\log \left(k^{2}(\phi)\right), \phi \in(-\pi, 0) \cup(0, \pi)
$$

(since $k$ is even, the boundary condition is well-defined). Then $u$ is bounded in $O$ and assumes its boundary values continuously on $c \backslash\{0\}$. Let $v$ be a harmonic conjugate for $u$ in $O$ and set $g=\exp (u+i v)$. Then $g$ extends continuously to $c \backslash\{0\}$ and in particular $|g(\lambda)| \rightarrow k^{2}(\phi)$ as $\lambda \rightarrow h^{2}(\phi) \in c \backslash\{0\}$.

The function $\lambda / g(\lambda)$ is nonvanishing on $O$, so we may choose a branch of the square root and define, for each fixed $\tau \in[-\pi, \pi)$ and $\lambda \in O$,

$$
G_{\tau}(\lambda)=\left(e^{2 i \tau} g(\lambda), e^{-i \tau} \sqrt{\frac{\lambda}{g(\lambda)}}\right) .
$$

\section{Theorem 4.1.}

$$
\widehat{\mathcal{K}}=\left\{G_{\tau}(\lambda): \tau \in[-\pi, \pi), \lambda \in \bar{O} \backslash\{0\}\right\} \cup \mathcal{D}_{0},
$$

and $\widehat{\mathcal{K}} \backslash \mathcal{K}$ has topological dimension equal to three.

Proof. Suppose that $p=\left(z_{0}, w_{0}\right) \in \widehat{\mathcal{K}}$ and $z_{0} w_{0}^{2}=\lambda_{0} \in c \backslash\{0\}$. If $\lambda_{0}=h^{2}(\phi)$, we claim that $p \in C_{\phi}$. For if $p \notin C_{\phi}$, by the polynomial convexity of $C_{\phi}$ we may choose a polynomial $Q$ with $Q(p)=1$ and $|Q|<1$ on $C_{\phi}$. We can also choose $f \in P(\bar{O})$ with $f\left(\lambda_{0}\right)=1$ and $|f|<1$ on $c \backslash\left\{\lambda_{0}\right\}$. Repeating the argument in Lemma 3.2 with $z f^{n}(w)$ replaced by $Q(z, w) f^{n}\left(z w^{2}\right)$, we arrive at a contradiction, proving the claim. The same argument with $\mathcal{D}_{0}$ replacing $C_{\phi}$ shows that $\left\{(z, w) \in \widehat{\mathcal{K}}: z w^{2}=0\right\}=\mathcal{D}_{0}$.

If as above $\lambda_{0}=h^{2}(\phi) \in c \backslash\{0\}$, then for some real $\sigma$,

$$
\lim _{\lambda \in O \rightarrow \lambda_{0}} G_{\tau}(\lambda)=\left(e^{2 i \tau} e^{i \sigma} k^{2}(\phi), \pm e^{-i \tau} \frac{h(\phi)}{e^{i \sigma / 2} k(\phi)}\right)=\mathcal{F}(\tau+\sigma / 2, \pm \phi) \in \mathcal{K},
$$

where the sign depends on the choice of square root. Therefore, after precomposing with a conformal map from $\mathbb{D}$ to $O$, we see that the image of $\lambda \mapsto G_{\tau}(\lambda)$ is an $H^{\infty}$ disk with boundary in $\mathcal{K}$, for each $\tau$, and so $\left\{G_{\tau}(\lambda): \tau \in[-\pi, \pi), \lambda \in \bar{O} \backslash\{0\}\right\} \subset \widehat{\mathcal{K}}$.

It remains to show that if $(z, w) \in \widehat{\mathcal{K}} \backslash \mathcal{K}$ and $z w^{2}=\lambda \in O$, then $(z, w)=$ $G_{\tau}(\lambda)$ for some $\tau$. We apply Wermer's Theorem (see section 3) with $A=P(\mathcal{K})$, $\pi(z, w)=z w^{2}, f(z, w)=z$ and $f(z, w)=w$ respectively to obtain functions $\psi_{1}, \psi_{2}$ subharmonic and bounded above in $O$, defined by

$$
\begin{aligned}
& \psi_{1}(\lambda)=\log \left(\max \left\{|z|:(z, w) \in \widehat{K}, z w^{2}=\lambda\right\}\right), \\
& \psi_{2}(\lambda)=\log \left(\max \left\{|w|:(z, w) \in \widehat{K}, z w^{2}=\lambda\right\}\right) .
\end{aligned}
$$

Arguments similar to those in Lemma 3.4 show that

$$
\psi_{1}(\lambda)=\log |g(\lambda)|, \psi_{2}(\lambda)=\log \left|\frac{\lambda}{g(\lambda)}\right|^{1 / 2}
$$

for all $\lambda \in O$. If $(z, w) \in \widehat{K}$ and $z w^{2}=\lambda \in O$, we therefore have

$$
|z| \leq|g(\lambda)|,|w| \leq\left|\frac{\lambda}{g(\lambda)}\right|^{1 / 2} .
$$


Therefore

$$
|\lambda|=\left|z w^{2}\right| \leq|\lambda|
$$

implying that both inequalities in (4.2) are in fact equalities. We may thus write $z=e^{2 i \tau} g(\lambda)$ for some $\tau$, and then the fact that $z w^{2}=\lambda$ implies $w=e^{-i \tau} \sqrt{\lambda / g(\lambda)}$ (replacing $\tau$ by $\tau+\pi$ if necessary to match the sign of the root). Therefore $(z, w)=$ $G_{\tau}(\lambda)$. This completes the proof that $\widehat{\mathcal{K}}=\left\{G_{\tau}(\lambda): \tau \in[-\pi, \pi), \lambda \in \bar{O} \backslash\{0\}\right\} \cup \mathcal{D}_{0}$. Note that $\widehat{\mathcal{K}} \backslash \mathcal{K}$ is the union of $\left\{G_{\tau}(\lambda): \tau \in[-\pi, \pi), \lambda \in O\right\}$ and $D_{0} \backslash\{(z, 0)$ : $\left.|z|=(a \pm b)^{2}\right\}$. It is not hard to check that $(\tau, \lambda) \mapsto G_{\tau}(\lambda)$ is locally one-to-one on $[-\pi, \pi) \times O$, and so $\widehat{\mathcal{K}} \backslash \mathcal{K}$ has topological dimension three.

\section{REFERENCES}

[1] H. Alexander and J. Wermer, Polynomial Hulls with Convex Fibers, Math. Ann. 271 (1985), 99-109. MR779607 (86i:32025)

[2] J. Anderson, On an Example of Ahern and Rudin, Proc. A.M.S. 116, no. 3 (1992), 695-699. MR.1129870 (93c:32021)

[3] A. Derdzinski and T. Januszkiewicz, Totally Real Immersions of Surfaces, Trans. A.M.S. 362, no. 1 (2010), 53-115. MR2550145 (2010j:53108)

[4] J. Duval and D. Gayet, Riemann Surfaces and Totally Real Tori, arXiv:math.cv/0910.2139

[5] F. Forstnerič, Complex Tangents of Real Surfaces in Complex Surfaces, Duke Math. J. 67, no. 2 (1992), 353-376. MR1177310 (93g:32025)

[6] F. Forstnerič, Analytic disks with boundaries in a maximal real submanifold of $\mathbb{C}^{2}$, Ann. Inst. Fourier (Grenoble) 37, no. 1 (1987), 1-44. MR0894560 (88j:32019)

[7] J. Garnett and D. Marshall, Harmonic Measure, Cambridge University Press, 2005. MR2150803 (2006g:31002)

[8] P. Koosis, Introduction to $H_{p}$ Spaces, second edition, Cambridge University Press, 1998. MR1669574 (2000b:30052)

[9] T. Ransford, Potential Theory in the Complex Plane, Cambridge University Press, 1995. MR.1334766 (96e:31001)

[10] W. Rudin, Totally Real Klein Bottles in $\mathbb{C}^{2}$, Proc. A.M.S. 82, no. 4 (1981), 653-654. MR614897 (82i:32012)

[11] E. L. Stout, Polynomial Convexity, Prog. in Math. 261, Birkhäuser, 2007. MR.2305474 (2008d:32012)

[12] J. Wermer, Subharmonicity and Hulls, Pac. J. Math. 58, no. 1 (1975), 283-290. MR0393567 $(52: 14376)$

Department of Mathematics and Computer Science, College of the Holy Cross, Worcester, Massachusetts 01610

E-mail address: anderson@mathcs.holycross.edu 